\title{
Quality of Life in Men With Prostate Cancer Randomly Allocated to Receive Docetaxel or Abiraterone in the STAMPEDE Trial
}

\author{
Hannah L. Rush, MBChB ${ }^{1}$; Laura Murphy, PhD ${ }^{1}$; Alicia K. Morgans, MD²; Noel W. Clarke, ChM ${ }^{3}$; Adrian D. Cook, MSc ${ }^{1}$; \\ Gerhardt Attard, MD, PhD ${ }^{4}$; Archie Macnair, MBBS ${ }^{1,5}$; David P. Dearnaley, MD ${ }^{6}$; Christopher C. Parker, MD ${ }^{6}$; J. Martin Russell, MBChB; \\ Silke Gillessen, MD ${ }^{8}$; David Matheson, PhD ${ }^{9}$; Robin Millman ${ }^{1}$; Christopher D. Brawley, MSc ${ }^{1}$; Cheryl Pugh, BSc ${ }^{1}$; \\ Jacob S. Tanguay, MBChB ${ }^{10}$; Robert J. Jones, PhD7; John Wagstaff, MD ${ }^{11}$; Sarah Rudman, PhD ${ }^{5}$; Joe M. O'Sullivan, MD ${ }^{12}$; \\ Joanna Gale, BM, DM ${ }^{13}$; Alison Birtle, MD ${ }^{14,15}$; Andrew Protheroe, PhD ${ }^{16}$; Emma Gray, MBChB ${ }^{17}$; Carla Perna, MD ${ }^{18}$; \\ Shaun Tolan, $\mathrm{MBBCh}^{19}$; Neil McPhail, $\mathrm{MBChB}^{20}$; Zaf I. Malik, MBChB ${ }^{19}$; Salil Vengalil, MBBS ${ }^{21}$; David Fackrell, MBChB ${ }^{22}$;

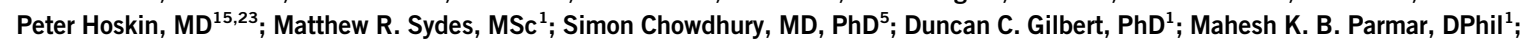 \\ Nicholas D. James, $\mathrm{PhD}^{6}$; and Ruth E. Langley, $\mathrm{PhD}^{1}$
}

PURPOSE Docetaxel and abiraterone acetate plus prednisone or prednisolone (AAP) both improve survival when commenced alongside standard of care (SOC) androgen deprivation therapy in locally advanced or metastatic hormone-sensitive prostate cancer. Thus, patient-reported quality of life $(\mathrm{QOL})$ data may guide treatment choices.

METHODS A group of patients within the STAMPEDE trial were contemporaneously enrolled with the possibility of being randomly allocated to receive either docetaxel + SOC or AAP + SOC. A mixed-model assessed QOL in those who had completed at least one QLQ-C30 + PR25 questionnaire. The primary outcome measure was difference in global-QOL (QLQ-C30 Q29\&30) between patients allocated to docetaxel + SOC or AAP + SOC over the 2 years after random assignment, with a predefined criterion for clinically meaningful difference of $>4.0$ points. Secondary outcome measures included longitudinal comparison of functional domains, pain, and fatigue, plus global-QOL at defined timepoints.

RESULTS Five hundred fifteen patients (173 docetaxel + SOC and 342 AAP + SOC) were included. Baseline characteristics, proportion of missing data, and mean baseline global-QOL scores (docetaxel + SOC 77.8 and $\mathrm{AAP}+\mathrm{SOC} 78.0$ ) were similar. Over the 2 years following random assignment, the mean modeled global-QOL score was +3.9 points $(95 \% \mathrm{Cl},+0.5$ to $+7.2 ; P=.022)$ higher in patients allocated to AAP + SOC. Global-QOL was higher for patients allocated to AAP + SOC over the first year ( +5.7 points, $95 \% \mathrm{Cl},+3.0$ to $+8.5 ; P<.001$ ), particularly at $12(+7.0$ points, $95 \% \mathrm{Cl},+3.0$ to $+11.0 ; P=.001)$ and 24 weeks ( +8.3 points, $95 \% \mathrm{Cl},+4.0$ to $+12.6 ; P<.001)$.

CONCLUSION Patient-reported QOL was superior for patients allocated to receive AAP + SOC, compared with docetaxel + SOC over a 2-year period, narrowly missing the predefined value for clinical significance. Patients receiving AAP + SOC reported clinically meaningful higher global-QOL scores throughout the first year following random assignment.

ASSOCIATED

CONTENT

Appendix

Protocol

Author affiliations

and support

information (if

applicable) appear

at the end of this

article.

Accepted on

October 1, 2021 and

published at

ascopubs.org/journal/

jco on November 10 ,

2021: Dol https://doi.

org/10.1200/JC0.21.

00728

J Clin Oncol 00. $\odot 2021$ by American Society of Clinical Oncology

\section{INTRODUCTION}

Over the past 5 years, several therapies have been approved in combination with androgen deprivation therapy (ADT) for the treatment of metastatic hormone-sensitive prostate cancer (HSPC). ${ }^{1}$ STAMPEDE (Systemic Therapy for Advanced or Metastatic Prostate Cancer: Evaluation of Drug Efficacy; ClinicalTrials.gov identifier: NCT00268476) is a multiarm, multistage platform trial assessing different treatment regimens given alongside or instead of long-term ADT. Data from STAMPEDE and other trials have demonstrated that treatment with docetaxel or with abiraterone acetate plus prednisone or prednisolone
(AAP) started concurrently with long-term ADT significantly prolongs overall survival when compared with ADT alone..$^{2-7}$

Patients with prostate cancer report a willingness to compromise maximal survival to achieve better quality of life (QOL). ${ }^{8}$ Although ADT is associated with multiple side effects that negatively affect $\mathrm{QOL},{ }^{9}$ the impact on $\mathrm{QOL}$ from additional treatment with docetaxel or AAP has not been directly compared.

There have been no prospectively randomized trials directly comparing docetaxel to AAP in patients with HSPC. However, between November 2011 and March 


\section{CONTEXT}

\section{Key Objective}

Docetaxel and abiraterone acetate plus prednisone or prednisolone (AAP) both prolong overall survival for men with hormone-sensitive prostate cancer starting long-term androgen deprivation therapy, with no clear evidence either treatment is superior. This analysis aimed to determine if differences in quality of life (QOL) scores over a 2-year period in patients allocated to receive docetaxel + standard of care (SOC) or AAP + SOC within the STAMPEDE trial were clinically meaningful. This study is the only published analysis of QOL comparing docetaxel + SOC and AAP + SOC from a contemporaneously randomly assigned group of patients.

\section{Knowledge Generated}

Global-QOL scores over the first 2 years were significantly higher in patients allocated to AAP + SOC compared with docetaxel $+\mathrm{SOC}$, although they did not meet the threshold to be clinically meaningful. Several secondary analyses favored AAP + SOC, particularly in the first year of treatment where results exceeded the clinically meaningful threshold.

\section{Relevance}

Understanding the difference in QOL scores will help clinicians and patients choose between docetaxel + SOC or $\mathrm{AAP}+\mathrm{SOC}$ as upfront treatment for hormone-sensitive prostate cancer.

2013, a group of patients within STAMPEDE were contemporaneously enrolled with the potential to be randomly allocated to receive either six cycles of docetaxel with daily prednisolone during chemotherapy + standard of care (SOC) or daily AAP + SOC (Appendix Fig A1, online only), creating an opportunity for comparative studies. Although not directly preplanned (in terms of power, type 1 error, and sample size), an analysis of survival outcomes in these patients found no evidence of difference in overall survival. ${ }^{10}$ Here, we present a comparison of patient-reported QOL data from these patients contemporaneously randomly allocated to receive docetaxel + SOC or AAP + SOC within the STAMPEDE platform.

\section{METHODS}

The design of STAMPEDE has been described previously. ${ }^{11}$ In brief, STAMPEDE opened to recruitment in October 2005 with patients randomly allocated to one of five treatment regimens including docetaxel $+\mathrm{SOC}$, or a shared control arm (SOC alone). A new research arm AAP + SOC opened to recruitment in November 2011, using the ongoing shared control arm and partially overlapping with recruitment to the docetaxel + SOC arm until March 2013, when the docetaxel + SOC arm closed to recruitment. Patients with high-risk locally advanced or metastatic HSPC with a plan to start long-term ADT were eligible for this analysis if they had been randomly allocated to either docetaxel + SOC or AAP + SOC in STAMPEDE during this overlapping period (November 2011-March 2013; Appendix Fig $\mathrm{A} 1$ ), and had completed at least one QOL questionnaire at any timepoint. Allocation to a treatment group used minimization with a random element across predefined stratification factors and a random assignment ratio of 1:2:2 (docetaxel + SOC, AAP + SOC, or control group; Fig 1).
National regulatory and ethics committees approved the Protocol (online only), participating hospital sites obtained local approval, and participants provided written informed consent.

\section{Intervention}

All treatments were open-label. SOC ADT for patients with nonmetastatic disease was a minimum of 2 years and patients with metastatic disease were planned for lifelong treatment. Therefore, all patients in this analysis had similar ADT exposure. Prostate \pm pelvic lymph node-directed radiotherapy was at the treating physicians' discretion.

Patients received SOC plus either docetaxel $\left(75 \mathrm{mg} / \mathrm{m}^{2} \mathrm{IV}\right.$ ) administered 3 weekly for up to six cycles, with $5 \mathrm{mg}$ prednisolone BD continuously, or abiraterone acetate (1,000 mg) orally plus $5 \mathrm{mg}$ prednisolone or prednisone both daily. AAP was continued for 2 years in patients with nonmetastatic disease and permitted until biochemical, radiologic, and clinical progression occurred in those with metastatic disease.

\section{Questionnaires Used and Scoring Method}

All patients who provided consent were eligible to join the QOL substudy. The EORTC QLQ-C30 version 3.0 questionnaire $^{12}$ with the prostate cancer-specific module PR25 ${ }^{13}$ was completed at baseline and at trial follow-up visits: 6-weekly for the first 6 months, 12-weekly until 2 years, 6-monthly until 5 years, and annually thereafter.

Questionnaires were scored as per EORTC guidelines. ${ }^{14}$ Questions from the global-QOL scale, functional domain scales, and symptom scales were considered with all scores standardized to a value between 0 and 100 .

Thresholds to define a clinically meaningful difference (CMD) in QOL scores were derived from a previous metaanalysis and expert panel review. ${ }^{15}$ The CMD threshold 


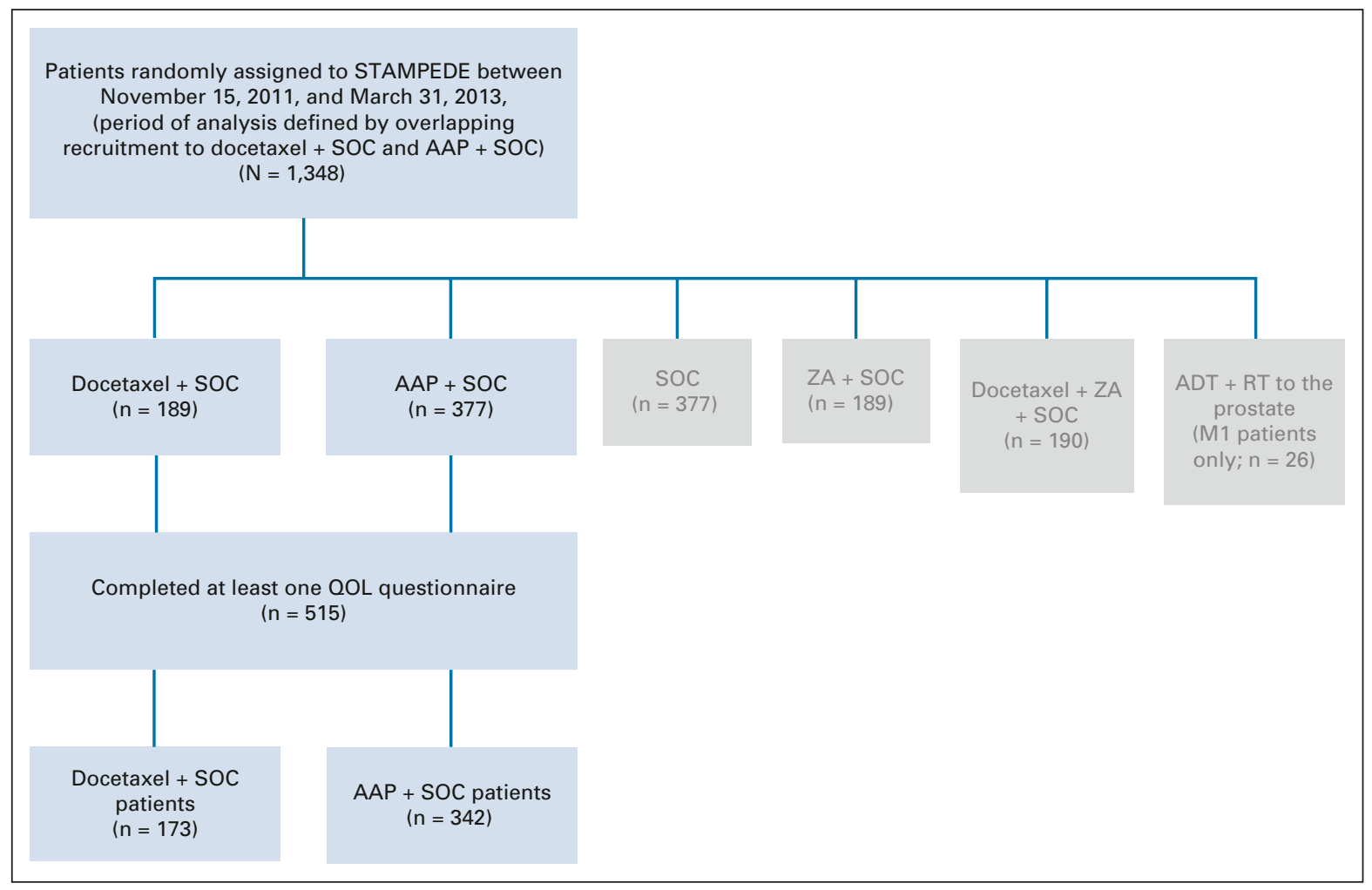

FIG 1. CONSORT diagram. AAP, abiraterone acetate plus prednisolone or prednisone; ADT, androgen deprivation therapy; M1, Metastatic prostate cancer; QOL, quality of life; RT, radiotherapy; SOC, standard of care; ZA, zoledronic acid.

distinguishes between differences in scores that are statistically significant, yet patients are unlikely to notice from differences likely to reflect a tangible benefit or detriment. The CMD threshold varies according to domains tested, with the CMD threshold for global-QOL defined as a difference $>4$ points (Appendix Table A1, online only).

The results are presented with the docetaxel + SOC score subtracted from the AAP + SOC. Hence, a positive value indicates the AAP + SOC group score is higher, and a negative value indicates the docetaxel + SOC group score is higher. Higher scores indicate better QOL, except for symptom scales where a higher score indicates a greater burden of symptoms or worse QOL.

\section{Statistical Methods}

Patients were censored at the start of second-line treatment or death. All data were analyzed using the intention-to-treat principle. The sample size was determined by the main STAMPEDE trial design with no dedicated calculation for the QOL analyses. The trial did not collect reasons for missing questionnaires, and missing data were not imputed.

Longitudinal, repeated-measures analyses used mixedeffects models with time and treatment interaction, unstructured covariance, and patient-level random effects. Cross-sectional analyses used linear regression, adjusted for baseline score.

\section{Outcome Measures}

The primary outcome measure was difference in globalQOL scores (QLQ-C30 Q29: "How would you rate your overall health during the last week?" and Q30: "How would you rate your overall quality of life during the last week?") over the first 2 years from random assignment between patients allocated to receive docetaxel $+\mathrm{SOC}$ or $\mathrm{AAP}+\mathrm{SOC}$, using repeated-measures analysis.

Secondary outcome measures were difference over 2 years in each functional domain (physical, emotional, role, cognitive, and social) and two symptoms (pain and fatigue), comparing docetaxel + SOC with AAP + SOC using repeated-measures analysis. Additionally, global-QOL scores were compared between groups using crosssectional analysis at weeks 12, 24, 48, and 104.

Exploratory analyses included difference in global-QOL between docetaxel + SOC and AAP + SOC over the first year, difference in patients with metastatic or nonmetastatic disease, and postprogression. QLQ-C30 summary scores ${ }^{14}$ (a validated summary score that looks across all domains) were compared. Finally, global-QOL scores in the control group patients were compared with each treatment group.

\section{RESULTS}

Between November 15, 2011, and March 31, 2013, 566 patients were randomly allocated to receive either 
docetaxel + SOC or AAP + SOC within STAMPEDE. Of these, 515 patients (docetaxel + SOC $n=173$ and AAP + SOC $n=342$ ) completed at least one QOL questionnaire and were included in this analysis (Fig 1). The data for these analyses were frozen on December 3, 2019.

Baseline characteristics were similar between the groups, including pain at baseline and validated prognostic variables such as age, stage, and performance status (Table 1). Baseline characteristics of the patients were similar to those of the larger docetaxel $+\mathrm{SOC}$ and AAP + SOC groups previously reported. 2,3 Baseline global-QOL scores were also similar: docetaxel + SOC 77.8 (standard deviation 20.0) and AAP + SOC 78.0 (standard deviation 19.3).

Baseline QOL questionnaires were received from $89 \%$ of patients in both treatment groups. Questionnaires were defined as expected at each follow-up visit, provided the patient was not censored. Overall, during the 2-year analysis period, the proportion of expected questionnaires received was $67 \%$ in the docetaxel + SOC group and $75 \%$ in the AAP + SOC group. There was no evidence that missing data correlated with baseline characteristics including age or metastatic status. The proportion of missing forms is shown in Appendix Table A2 and Figure A2 (online only).

Over the 2 years following random assignment, 34 of 171 participants (19.9\% - two patients only providing data postprogression) in the docetaxel + SOC group and 71 of 342 participants $(20.8 \%)$ in the AAP + SOC group were censored for starting second-line treatment or death.

Failure-free survival (FFS; defined as time from random assignment to the first of either biochemical, lymph node, or distant metastatic progression; or prostate cancerspecific death) was $64.1 \%$ within the 2 -year analysis period for the docetaxel + SOC group (58 events, 25 patients given subsequent treatment with life-prolonging therapy). In the AAP + SOC group, FFS was 76.4\% (80 events, 41 patients with subsequent life-prolonging therapy).

\section{Treatment}

In the docetaxel + SOC group, 160 of $173(92.4 \%)$ started docetaxel, 137 of 173 (79.2\%) completed six cycles, whereas 21 of 160 patients stopped early because of excess toxicity, and 2 of 160 for other nonspecified reasons.

In the AAP + SOC group, 340 of 342 (99.0\%) participants started AAP. Median time on treatment was 697 days (interquartile range, 332-751 days) with time on treatment censored for each patient at the final QOL questionnaire contributing to the analysis (therefore likely shorter than actual time on treatment). Within the first 2 years, 263 of $342(77.0 \%)$ participants were still taking AAP when the last QOL questionnaire was received, and 11 patients stopped AAP because of toxicity.
Two-year longitudinal analysis. Over the 2-year period after random assignment, the mean modeled global-QOL score was 74.5 (SE, 1.0) among the AAP + SOC group, and 70.6 (SE, 1.4) among the docetaxel + SOC group, a difference of +3.9 points $(95 \% \mathrm{Cl}, 0.5$ to $7.2 ; P=.02)$ favoring AAP + SOC. This was statistically significant, but just failed to reach the predefined threshold of $>4$ points denoting a CMD (Fig 2).

QLQ-C30 functional domains. In the 2 years following random assignment, there was a statistically significant and CMD favoring AAP + SOC in the domain assessing social function ( $+5.0,95 \% \mathrm{Cl}, 1.3$ to $8.7 ; P=.008$ ). Statistically significant differences in physical $(+4.5,95 \% \mathrm{Cl}, 1.3$ to 7.7; $P=.006)$ and role function $(+5.8,95 \% \mathrm{Cl}, 1.6$ to 9.9 ; $P=.006)$ favoring AAP + SOC did not meet the CMD threshold (Appendix Table A1). There was no evidence of difference in cognitive function $(-0.2,95 \% \mathrm{Cl},-3.4$ to 3.0; $P=.920$ ) or emotional function ( $+0.2,95 \% \mathrm{Cl},-3.1$ to 3.6; $P=.885 ;$ Fig 3).

QLQ-C30 symptoms. Over 2 years after random assignment, the difference was -3.9 points $(95 \% \mathrm{Cl},-7.7$ to -0.1 ; $P=.042)$ in reported fatigue scores and -6.3 points (95\% $\mathrm{Cl},-10.0$ to $-2.6 ; P<.001)$ for pain scores, both favoring AAP + SOC (as higher scores indicate worse symptoms). This was only clinically meaningful for pain scores (Fig 4).

Cross-sectional analysis. At 12 weeks and 24 weeks, the difference in global-QOL scores was +7.0 points $(95 \% \mathrm{Cl}$, 3.0 to $11.0 ; P=.001)$ and +8.3 points $(95 \% \mathrm{Cl}, 4.0$ to 12.6; $P<.001$ ) favoring the AAP + SOC group, respectively. At 48 weeks, there was no apparent difference in global-QOL scores, +1.8 points $(95 \% \mathrm{Cl},-2.5$ to 6.0 ; $P=.412$ ), whereas at 104 weeks, the difference was +4.8 points $(95 \% \mathrm{Cl}, 0.0$ to $9.5 ; P=.048)$ favoring the $\mathrm{AAP}+\mathrm{SOC}$ group. Thus, the difference in global-QOL met the CMD threshold at 12, 24, and 104 weeks.

A summary of all secondary analyses performed is given in Table 2. A sensitivity analysis comparing the results using censored data versus all available data did not change the conclusions of these analyses.

Longitudinal analysis for global-QOL (year 1). Using the mixed-model approach, the difference in global-QOL scores over 1 year following random assignment was +5.7 points $(95 \% \mathrm{Cl}, 3.0$ to $8.5 ; P<.001)$ with patients in the AAP + SOC group reporting a statistically significantly and clinically meaningful superior QOL compared with those in the docetaxel + SOC group.

Nonmetastatic and metastatic disease. Longitudinal analyses of global-QOL were repeated separately for patients with nonmetastatic and metastatic disease. There was a similar proportion of patients in each treatment group with nonmetastatic disease (71 of 173 [41.0\%] for docetaxel + SOC and 137 of 342 [40.1\%] AAP + SOC) and metastatic disease (102 of 173 [58.9\%] docetaxel + SOC and 205 of 342 [59.9\%] AAP + SOC). For patients with 
TABLE 1. Baseline Characteristics of Patients Included in This Analysis, Comparing Patients Allocated to Receive SOC Alone, Docetaxel + SOC, or AAP + SOC During the Period of Overlapping Recruitment to These Treatments

Characteristic

SOC Alone $(n=343)$

Docetaxel + SOC $(n=173)$

$A A P+\operatorname{SOC}(n=342)$

Age at random assignment, years

Median (IQR)

$66(62-71)$

$66(62-71)$

$66(61-70)$

WHO performance status, No. (\%)

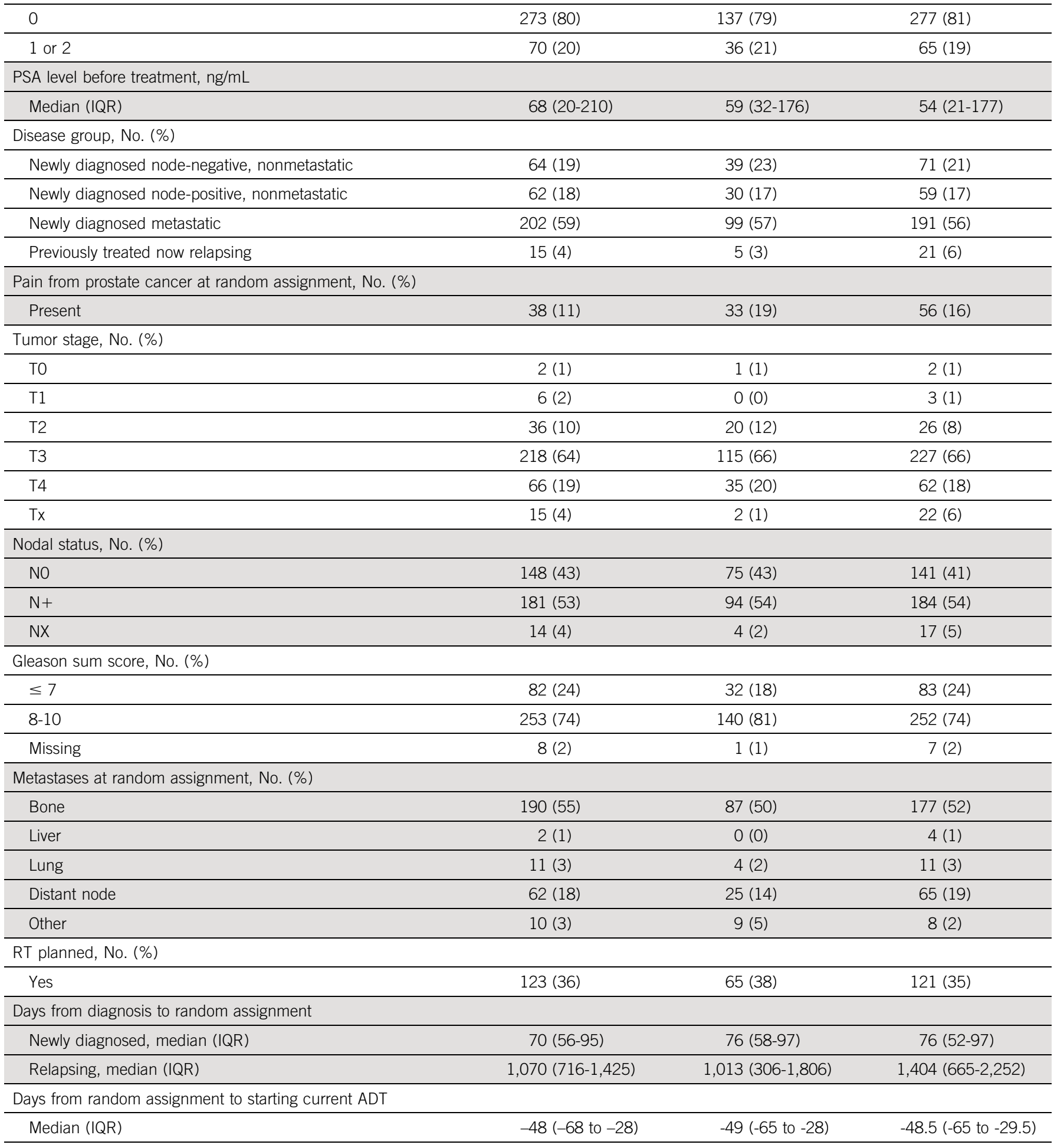

(continued on following page) 
TABLE 1. Baseline Characteristics of Patients Included in This Analysis, Comparing Patients Allocated to Receive SOC Alone, Docetaxel + SOC, or AAP + SOC During the Period of Overlapping Recruitment to These Treatments (continued)

Characteristic

SOC Alone $(n=343)$

Docetaxel + SOC $(n=173)$

$A A P+\operatorname{SOC}(n=342)$

Prior hormone treatment, No. (\%)

\begin{tabular}{lccc}
\hline None & $330(96)$ & $169(98)$ & $330(96)$ \\
\hline LHRHa \pm short course AAs & $11(3)$ & $3(2)$ & $7(2)$ \\
\hline Antiandrogens alone & $2(1)$ & $1(1)$ & $5(1)$ \\
\hline
\end{tabular}

Length of prior hormone treatment, days

\begin{tabular}{|c|c|c|c|}
\hline Median (IQR) & $155(146-179)$ & $186.5(146-262)$ & $168(132-211)$ \\
\hline \multicolumn{4}{|c|}{ Months from previous hormone treatment and random assignment } \\
\hline Median (IQR) & $27(19-37)$ & $51(27-73)$ & $54(34-119)$ \\
\hline Orchidectomy & $1(<1)$ & $0(0)$ & $1(<1)$ \\
\hline LHRHa & 340 (99) & $173(100)$ & $341(100)$ \\
\hline Maximum androgen blockade & $1(<1)$ & $0(0)$ & $0(0)$ \\
\hline
\end{tabular}

Abbreviations: AA, antiandrogens; AAP, abiraterone acetate plus prednisone or prednisolone; ADT, androgen deprivation therapy; IQR, interquartile range; LHRHa, luteinising hormone-releasing hormone antagonist/agonist; PSA, prostate-specific antigen; RT, radiotherapy; SOC, standard of care.

metastatic disease, there was evidence of higher globalQOL scores in the AAP + SOC group ( +4.5 points, $95 \% \mathrm{Cl}$, 0.3 to $8.6 ; P=.036)$, but no clear evidence of difference in scores in patients with nonmetastatic disease $(+3.0,95 \%$ $\mathrm{Cl},-2.4$ to 8.3; $P=.275 ;$ Appendix Fig A3, online only). An interaction test found no evidence that metastatic status

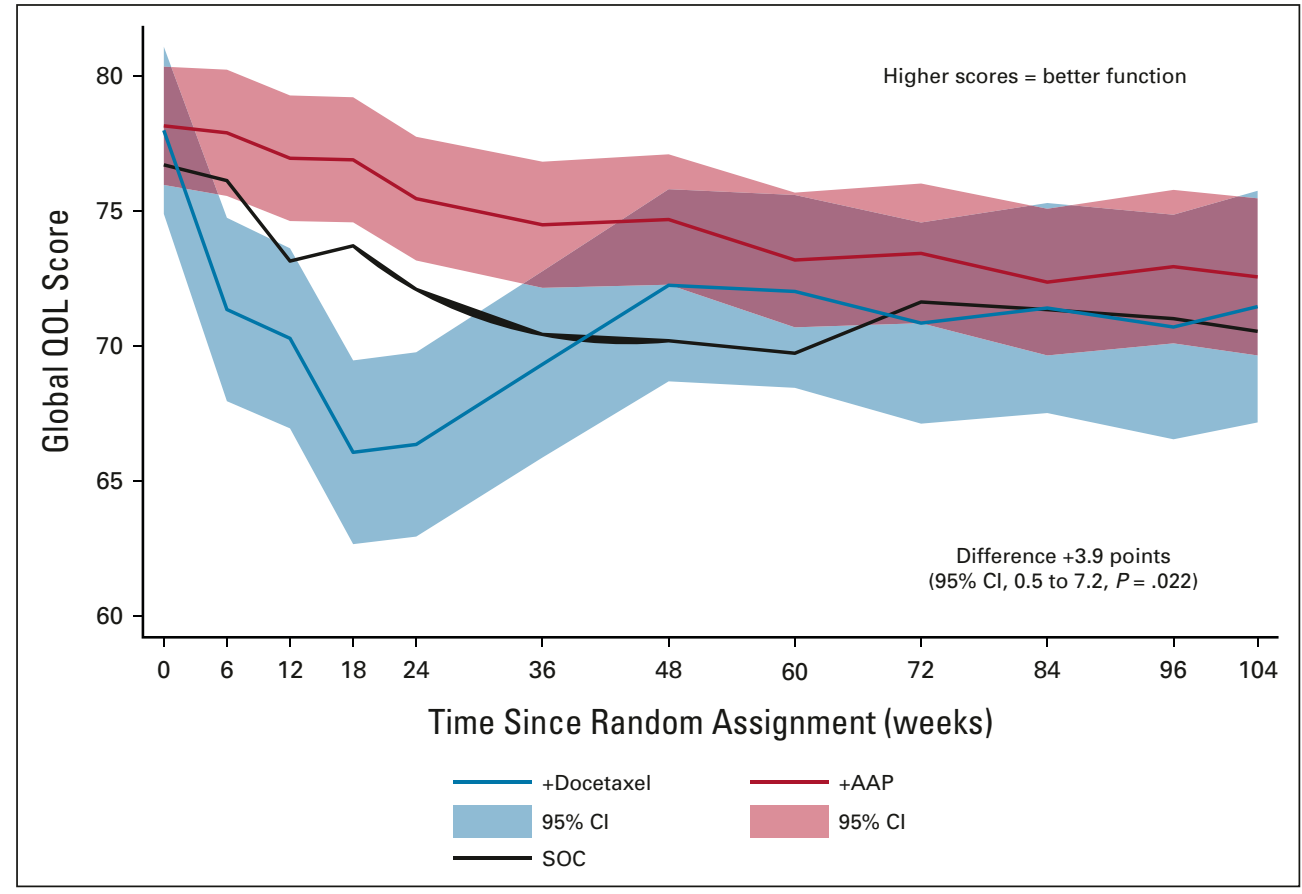

FIG 2. Longitudinal analysis using a mixed-model approach of global-QOL scores. The red line shows global-QOL score for patients treated with AAP + SOC, whereas the blue line shows global-QOL score for patients treated with docetaxel $+\mathrm{SOC}$ over the 2 years following random assignment. The red and blue shaded areas show the $95 \% \mathrm{Cl}$ around each line. The black line shows global-QOL for patients receiving SOC alone. Higher scores indicate better QOL. The difference is calculated by subtracting the docetaxel + SOC score from the AAP + SOC score; a positive difference represents a higher QOL for patients treated with AAP + SOC and negative difference represents a higher $\mathrm{QOL}$ for patients receiving docetaxel + SOC. All scores are corrected as per QLQ-C30 guidelines to range from 0 to 100. AAP, abiraterone acetate plus prednisone or prednisolone; QOL, quality of life; SOC, standard of care. 


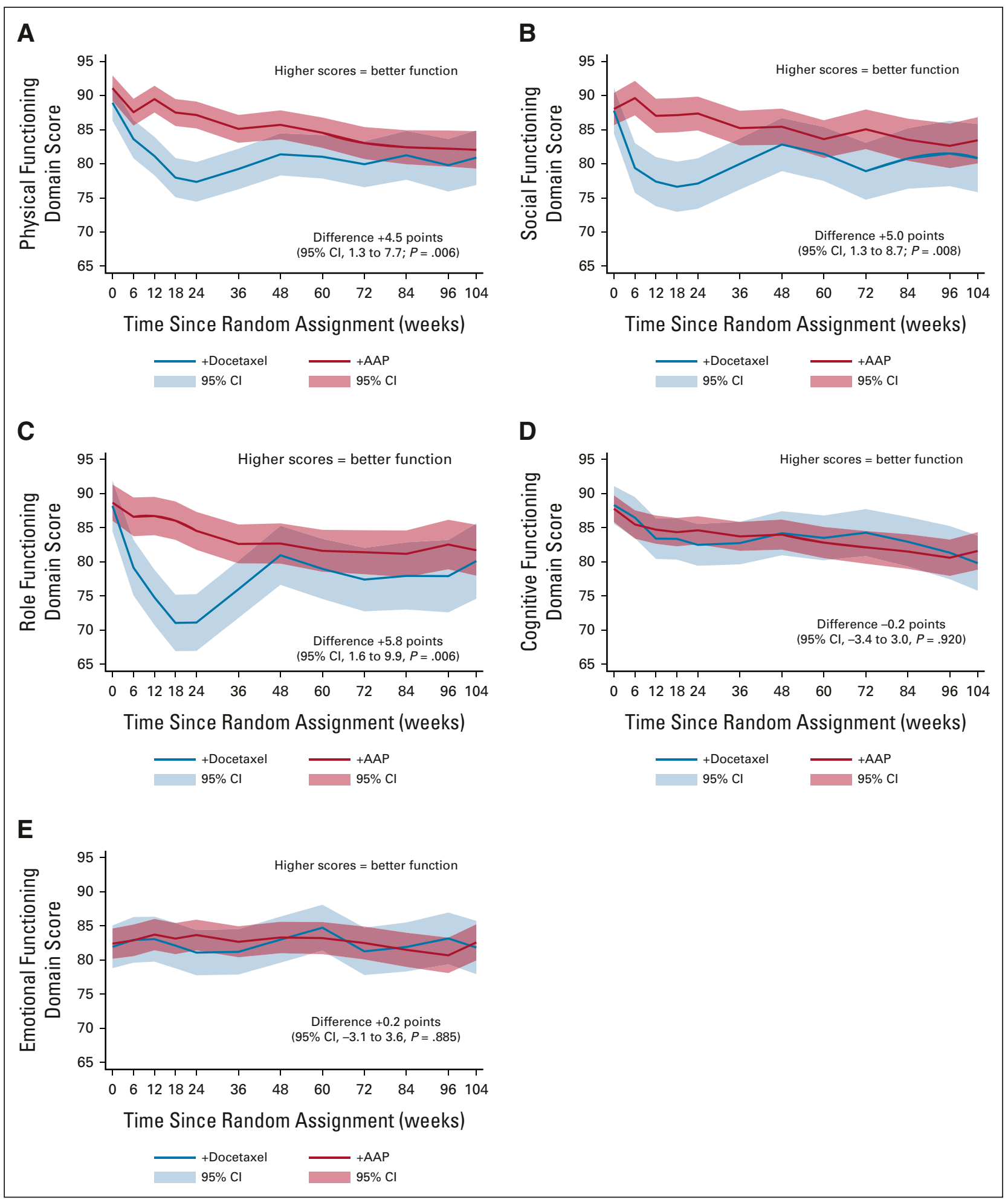

FIG 3. Longitudinal analysis showing QOL score for each functional domain over 2 years, separating scores for patients treated with docetaxel + SOC versus AAP + SOC. Higher scores indicate better quality of life. All scores are corrected as per QLQ-C30 guidelines to range from 0 to 100 . The difference over 2 years is calculated by subtracting the docetaxel + SOC score from the AAP + SOC score; positive scores represent a higher QOL for patients treated with AAP + SOC and negative scores indicate a higher QOL for patients treated with docetaxel + SOC: (A) physical functioning domain, (B) social functioning domain, (C) role functioning domain, (D) cognitive functioning domain, and (E) emotional functioning domain. AAP, abiraterone acetate plus prednisone or prednisolone; QOL, quality of life; SOC, standard of care. 
A

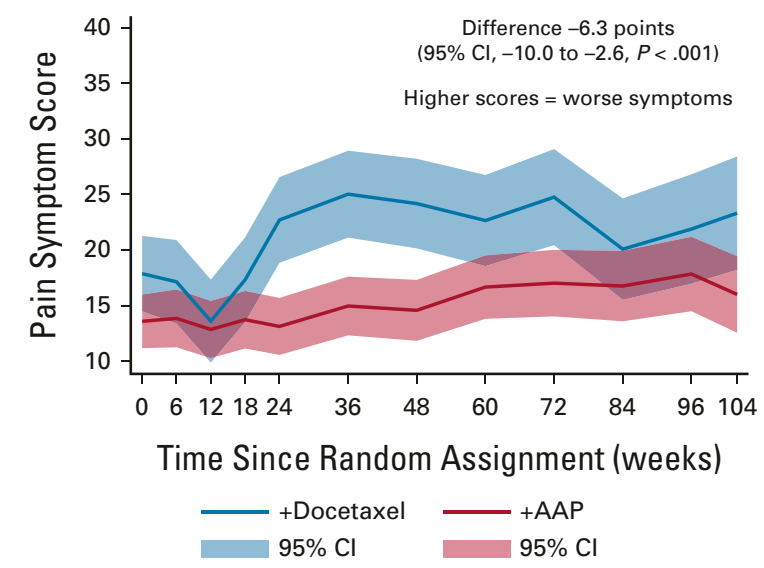

B

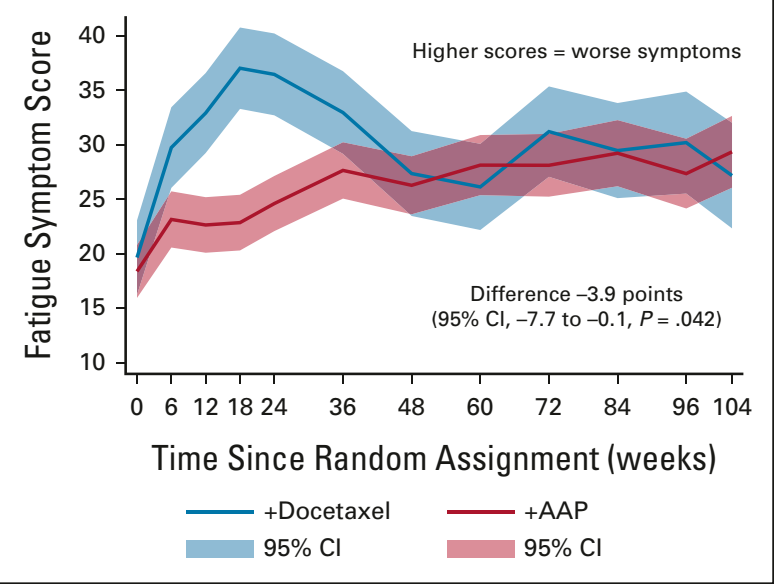

FIG 4. Longitudinal analysis showing QOL score for pain and fatigue symptoms over 2 years, separating scores for patients treated with docetaxel + SOC versus AAP + SOC. The difference over 2 years is calculated by subtracting the docetaxel + SOC score from the AAP + SOC score; a positive difference represents a higher symptom score for patients treated with AAP + SOC and a negative difference indicates a higher symptom score for patients receiving docetaxel + SOC. Higher scores indicate worse symptoms and quality of life. All scores are corrected as per QLQ-C30 guidelines to range from 0 to 100 : (A) pain symptoms and (B) fatigue symptoms. AAP, abiraterone acetate plus prednisone or prednisolone; QOL, quality of life; SOC, standard of care.

had a differential effect on difference between QOL scores in patients treated with docetaxel + SOC versus AAP + SOC (interaction $P=.701$ ).

QLQ-C30 summary scores. Over the 2-year period, the average QLQ-C30 summary score was 85.0 (SE, 0.7) with $\mathrm{AAP}+\mathrm{SOC}$ and 82.4 (SE, 1.0) with docetaxel + SOC, a difference of +2.6 points $(95 \% \mathrm{Cl}, 0.1$ to $5.1 ; P=.041)$ favoring AAP + SOC (Appendix Fig A4, online only).

QOL from the point of progression. A longitudinal analysis of global-QOL for 12 months postprogression included data from 58 of 173 (33.5\%) patients randomly assigned to docetaxel + SOC and 80 of 342 (23.4\%) patients AAP + SOC. There was no evidence of a difference in global-QOL scores following progression in this small group $(-4.9$ points; $95 \% \mathrm{Cl},-13.2$ to 3.4; $P=.247$; Appendix Fig A5, online only).

Comparison to Soc. There was no difference between global-QOL scores in the docetaxel + SOC group compared with control group $(-1.0$ points, $95 \% \mathrm{Cl},-4.4$ to +2.3 ; $P=.553)$, and higher scores with AAP + SOC compared with control, although this did not meet the CMD threshold (+2.9 points, $95 \% \mathrm{Cl}, 0.1$ to 5.6; $P=.040$ ).

\section{DISCUSSION}

During the first 2 years following random assignment, global-QOL scores were higher for patients allocated to receive $A A P+S O C$, although this did not meet the CMD threshold. However, there was a CMD between groups over the first year following random assignment, and at the 12-, 24-, and 104-week timepoints favoring AAP + SOC. Moreover, scores favored AAP + SOC over 2 years in the role domain (encompassing activities of daily living, mobility, and work capacity) and lower levels of pain.

Our analysis included more than 500 contemporaneously randomly assigned patients well matched for baseline characteristics. The trial collected QOL at multiple timepoints enabling a detailed longitudinal analysis. Although $\mathrm{QOL}$ is an important outcome measure for all interventional clinical trials ${ }^{16}$ with guidelines to standardize reporting, ${ }^{17}$ variations in trial design, tools, and statistical methods often prevent meaningful cross-trial comparisons. As far as the authors are aware, this is the only direct comparison of QOL reported by patients randomly assigned to receive docetaxel + SOC or AAP + SOC.

At 12 weeks, falling QOL scores in the docetaxel + SOC group almost certainly reflect toxicity from chemotherapy. By 24 weeks, docetaxel treatment is completed, with patients remaining on ADT alone. Our analysis showed lower QOL scores in the docetaxel + SOC group throughout the first year, suggesting persistent or slow-to-recover toxicity. This is likely to be an important factor contributing to difference in QOL scores.

From 12 weeks onward, patients treated with docetaxel + SOC reported higher pain scores, potentially contributing to worse global-QOL scores. This could reflect increased prevalence of persistent painful toxicities in the docetaxel + SOC group, such as peripheral neuropathy or chemotherapy-induced pain syndromes, or withdrawal of steroids given with chemotherapy.

It does not appear that higher QOL scores in the AAP + SOC group were because of differences in progression rates. FFS is largely driven by biochemical relapse, ie, not 
TABLE 2. Summary of Primary and Secondary Outcomes, Including Longitudinal Analysis of Global-QOL, Functional Scores and Symptoms, and CrossSectional Analysis of Global-QOL Scores at 12, 24, 48, and 104 Weeks

Model Estimate (SE)

\begin{tabular}{|c|c|c|c|c|c|}
\hline Outcome & SOC Alone & Docetaxel + SOC & $A A P+S O C$ & Difference (95\% Cl; AAP score minus docetaxel score) & $P$ \\
\hline \multicolumn{6}{|c|}{ Longitudinal analysis of QOL over 2 years } \\
\hline Global-QOL & $71.6(1.0)^{\mathrm{a}}$ & $70.6(1.4)$ & $74.5(1.0)$ & $+3.9(0.5$ to 7.2$)$ & .022 \\
\hline Physical & & $80.5(1.4)$ & $85.0(0.9)$ & +4.5 (1.3 to 7.7$)$ & .006 \\
\hline Social & & $80.2(1.6)$ & $85.3(1.0)$ & $+5.0(1.3$ to 8.7$)$ & .008 \\
\hline Emotional & & $82.4(1.4)$ & $82.6(1.0)$ & $+0.2(-3.1$ to 3.6$)$ & .885 \\
\hline Cognitive & & $83.4(1.3)$ & $83.2(0.9)$ & $-0.2(-3.4$ to 3.0$)$ & .920 \\
\hline \multicolumn{6}{|c|}{ Longitudinal analysis of symptoms over 2 years } \\
\hline Pain & & $21.7(1.6)$ & $15.4(1.1)$ & $-6.3(-10.0$ to -2.6$)$ & $<.001$ \\
\hline Fatigue & & $30.4(1.6)$ & $26.5(1.1)$ & $-3.9(-7.7$ to -0.1$)$ & .042 \\
\hline
\end{tabular}

\section{Mean (SD) Observed Values From Patients With QOL Data at Each Timepoint}

\begin{tabular}{|c|c|c|c|c|c|}
\hline \multirow[b]{2}{*}{ Time Point } & & \multirow{2}{*}{$\begin{array}{c}\text { Difference }(95 \% \mathrm{CI}) \text { Using Regression of Global-QOL Score by } \\
\text { Baseline Score and Treatment }\end{array}$} & \multirow[b]{2}{*}{$P$} \\
\hline & SOC Alone & Docetaxel + SOC & $A A P+S O C$ & & \\
\hline Baseline & 76.1 (19.3) & $77.8(20.0)$ & $78.0(19.3)$ & & \\
\hline 12 weeks & 73.8 (21.9) & $70.7(22.2)$ & $76.5(19.5)$ & +7.0 (3.0 to 11.0$)$ & .001 \\
\hline 24 weeks & $72.1(22.4)$ & $67.9(21.1)$ & $75.6(20.9)$ & +8.3 (4.0 to 12.6$)$ & $<.001$ \\
\hline 48 weeks & $71.5(21.0)$ & $74.4(20.2)$ & $75.5(20.4)$ & $+1.8(-2.5$ to 6.0$)$ & .412 \\
\hline No. of patients & 341 & 172 & $342^{b}$ & & \\
\hline
\end{tabular}

Abbreviations: AAP, abiraterone acetate plus prednisolone or prednisone; QOL, quality of life; SOC, standard of care; SD, standard deviation. ${ }^{a}$ Estimate for global-QOL in SOC group comes from a separate mixed model to the comparison model.

${ }^{b}$ Number of AAP + SOC patients contributing to the mixed models social, emotional, and cognitive scores is 341 .

invariably associated with disease-related symptoms. FFS was markedly worse in the control group ( $45 \%$ at 2 years) than either treatment groups, yet mean global-QOL scores over 2 years in the control group were not lower than the docetaxel + SOC group. Also, we censored patients at death or start of second-line treatment (docetaxel 20\%; AAP 21\%), a time more likely to be associated with diseaserelated symptoms. Comparing results with- and without censored patients did not alter the outcome of our analyses.

We did not find convincing evidence that differences in QOL scores for patients treated with docetaxel + SOC compared with AAP + SOC were different in metastatic versus nonmetastatic disease. This may warrant further analysis.

Postprogression, there was a nonsignificant trend toward lower absolute global-QOL in the AAP + SOC group compared with docetaxel + SOC, noting that median time to progression occurred later in the AAP + SOC group.
Understanding QOL postprogression is important, particularly as patients with advanced prostate cancer may have multiple lines of treatment. Patients treated with docetaxel first may subsequently receive AAP and vice versa; in key trials, $38 \%$ of patients treated initially with docetaxel + ADT went on to receive additional life-prolonging therapy, ${ }^{4}$ whereas $30 \%$ of patients initially treated with AAP + ADT received subsequent life-prolonging treatment. ${ }^{5} \mathrm{QOL}$ gains during first treatment may potentially be nullified by subsequent treatment, although improvement in diseaserelated symptoms for patients with more advanced disease may counterbalance toxicity from treatment. Indeed, although we saw QOL scores generally decline over time for all treatments, previous studies report improvement in $\mathrm{QOL}$ for many patients treated with docetaxel for castrationresistant prostate cancer. ${ }^{18,19}$ Maximizing QOL during firstline treatment may be more meaningful for a greater proportion of patients as time to the first progression is generally the longest, with progressively shorter periods following each 
subsequent treatment. Moreover, there is no benefit in reserving treatments with a better QOL profile for patients who become unfit for subsequent treatment.

Other HSPC QOL studies support our findings. CHAARTED, a randomized trial of patients with metastatic HSPC allocated to receive docetaxel + ADT or ADT-alone, found that $\mathrm{QOL}$ scores in patients treated with docetaxel + ADT were significantly lower (suggesting worse QOL) at 3 months, but higher at 12 months compared with patients treated with ADT, although neither difference met the clinically important threshold. ${ }^{20}$ LATITUDE, a randomized comparison of $A A P+A D T$ to ADT-alone in patients with high-risk newly diagnosed metastatic HSPC, found that patients treated with AAP + ADT reported better total QOL at all timepoints. ${ }^{21} \mathrm{~A}$ network meta-analysis of HSPC trials, comparing QOL in the first year of treatment with docetaxel or AAP, reported superior $\mathrm{QOL}$ in patients treated with $\mathrm{AAP}$, with the biggest difference seen at 3 months. ${ }^{22}$

We did not demonstrate a CMD in global-QOL over a 2-year period, although there was a statistically significant difference favoring treatment with AAP + SOC. We based our definition of $\mathrm{CMD}$ on proposed boundaries for comparing two groups with different interventions at a single timepoint, as these could be adapted as thresholds for the difference in scores between the two treatment groups compared over 2 years. ${ }^{15}$ However, the optimal thresholds for CMD in QOL scores between two groups of patients incorporating repeated measures over time has not been defined. Values for CMD are not standardized across different comparative methodologies, and thresholds vary between type and stage of cancer, the QOL tool used, or when assessing improvement versus deterioration. ${ }^{23}$ Extrapolating previous approaches to define CMD into our methodology may underestimate the importance of a difference that is small but persistent over an extended period.

\section{AFFILIATIONS}

${ }^{1}$ MRC Clinical Trials Units at University College London, London, United Kingdom

${ }^{2}$ Northwestern University, Chicago, IL

${ }^{3}$ The Christie and Salford Royal NHS Foundation Trusts, Manchester, United Kingdom

${ }^{4}$ UCL Cancer Institute, London, United Kingdom

${ }^{5}$ Guys and St Thomas' NHS Foundation Trust, London, United Kingdom

${ }^{6}$ Royal Marsden NHS Foundation Trust and the Institute of Cancer

Research, London, United Kingdom

${ }^{7}$ Institute of Cancer Sciences, University of Glasgow, Beatson West of

Scotland Cancer Centre, Glasgow, Scotland

${ }^{8}$ Oncology Institute of Southern Switzerland, Bellinzona, Switzerland

${ }^{9}$ University of Wolverhampton, Wolverhampton, United Kingdom

${ }^{10}$ Velindre Cancer Centre, Cardiff, Wales

${ }^{11}$ Swansea University College of Medicine, Swansea, United Kingdom

${ }^{12}$ Patrick G. Johnston Centre for Cancer Research, Queen's University

Belfast, United Kingdom

${ }^{13}$ Portsmouth Hospital University Trust, Portsmouth, United Kingdom
There are limitations of our analysis. Clinical trial populations do not always represent real-world patients. Nonetheless, STAMPEDE was designed to be a pragmatic trial with an open-label design and broad inclusion criteria. Open-label trial design may increase the risk of responder bias, although studies do not consistently support this for patient-reported outcomes. ${ }^{24-26}$ Disappointment and dropout from trial is a greater risk in patients allocated to SOC alone; responder bias should, therefore, be somewhat mitigated in our analysis comparing two interventional arms.

The PR25 questionnaire assesses symptoms associated with prostate cancer and side effects of ADT but has not been formally validated in the setting of current prostate cancer therapies. This may limit our ability to detect the impact of contemporary systemic treatments on QOL.

The reason for missing QOL questionnaires was not collected, and participation in the QOL substudy was optional. Missing data in QOL studies may occur randomly, arise from progression or death, or may be related to the unobserved health status of the participant. ${ }^{27}$ We found no evidence that missing data correlated with key clinical baseline characteristics, we censored patients at progression or death, and the quantity of missing data was similar between randomly assigned groups. The mixed-effects model, which is robust to missing-at-random-data, ${ }^{28,29}$ therefore remains appropriate.

In summary, this study demonstrates superior QOL for patients treated with AAP + SOC over the first year of treatment. Although the results did not meet the predefined clinically meaningful threshold over 2 years, our analyses consistently found that patients receiving AAP + SOC reported higher QOL scores. We believe the longitudinal analyses and associated figures illustrate these data clearly for patients and clinicians. This greater understanding of difference in $\mathrm{QOL}$ between these treatments will inform decisions about which upfront treatment to use alongside ADT.

\footnotetext{
${ }^{14}$ Rosemere Cancer Centre, Lancs Teaching Hospitals, Preston, United Kingdom

${ }^{15}$ University of Manchester, Manchester, United Kingdom

${ }^{16}$ Oxford University Hospitals NHS Foundation Trust, Oxford, United Kingdom

${ }^{17}$ Musgrove Park Hospital, Taunton, United Kingdom

${ }^{18}$ Royal Surrey Hospital Foundation Trust, Guildford, United Kingdom

${ }^{19}$ The Clatterbridge Cancer Centre NHS Foundation Trust, Liverpool, United Kingdom

${ }^{20}$ Raigmore Hospital, Inverness, Scotland

${ }^{21}$ University Hospital North Midlands NHS Trust, Stoke-on-Trent, United Kingdom

${ }^{22}$ University Hospital Birmingham, Birmingham, United Kingdom

${ }^{23}$ Mount Vernon Cancer Centre and University of Manchester,

Manchester, United Kingdom
} 


\section{CORRESPONDING AUTHOR}

Ruth E. Langley, PhD, MRC Clinical Trials Unit, Institute of Clinical Trials \& Methodology at University College London, 90 High Holborn, London WV1V 6LJ, United Kingdom; e-mail: ruth.langley@ucl.ac.uk.

\section{DISCLAIMER}

The funding bodies had no role in the conception or authorship of this publication.

\section{SUPPORT}

The STAMPEDE trial is sponsored by the UK Medical Research Council (MRC_MC_UU_12023/25) and conducted by the MRC Clinical Trials Unit at UCL. The trial is supported by CRUK (CRUK_A12459), the MRC the NIHR Clinical Research Network, and the Swiss Group for Clinical Cancer Research (SAKK). P.J.H. is supported by NIHR Manchester Biomedical Research Center. The trial also receives industry collaboration and support from Novartis, Sanofi-Aventis, Pfizer, Janssen, Clovis Oncology, and Astellas.

\section{CLINICAL TRIAL INFORMATION NCT00268476}

\section{AUTHORS' DISCLOSURES OF POTENTIAL CONFLICTS OF INTEREST}

Disclosures provided by the authors are available with this article at DOI https://doi.org/10.1200/JC0.21.00728.

\section{DATA SHARING STATEMENT}

The STAMPEDE trial data are held at MRC CTU at UCL, which encourages optimal use of data by using a controlled access approach to data sharing (http://www.ctu.mrc.ac.uk/our_research/datasharing/). Requests for data can be made at any time and can be initiated by contacting mrcctu.ctuenquiries@ucl.ac.uk or through the URL: http:// www.ctu.mrc.ac.uk/our_research/datasharing/application_process/. There is a formal application process, whereby the request will undergo review by the trial team, as well as independent researchers, to ensure that the proposed research is both ethical and has a strong scientific rationale. Data will not be released if it would compromise the ongoing trial. The specific data and associated documents to be shared will be dependent on the nature of the individual request and this will be documented in a formal data sharing agreement.

\section{AUTHOR CONTRIBUTIONS}

Conception and design: Hannah L. Rush, Noel W. Clarke, Archie Macnair, David P. Dearnaley, Silke Gillessen, David Matheson, Robin Millman, Christopher D. Brawley, Matthew R. Sydes, Duncan C. Gilbert, Mahesh K. B. Parmar, Nicholas D. James, Ruth E. Langley

Provision of study materials or patients: Gerhardt Attard, J. Martin Russell, Robert J. Jones, John Wagstaff, Sarah Rudman, Joe M. O'Sullivan, Andrew Protheroe, Emma Gray, Shaun Tolan, Zaf I. Malik, Matthew R. Sydes, Nicholas D. James

Collection and assembly of data: Christopher C. Parker, J. Martin Russell, Cheryl Pugh, Jacob S. Tanguay, Robert J. Jones, John Wagstaff, Sarah Rudman, Joe M. O'Sullivan, Joanna Gale, Alison Birtle, Andrew Protheroe, Emma Gray, Carla Perna, Shaun Tolan, Neil McPhail, David Fackrell, Peter Hoskin, Matthew R. Sydes, Nicholas D. James

Data analysis and interpretation: Hannah L. Rush, Laura Murphy, Alicia K. Morgans, Noel W. Clarke, Adrian D. Cook, Gerhardt Attard, David P. Dearnaley, Christopher C. Parker, J. Martin Russell, Silke Gillessen, David Matheson, Robin Millman, John Wagstaff, Joe M. O'Sullivan, Alison Birtle, Zaf I. Malik, Salil Vengalil, Peter Hoskin, Matthew R. Sydes, Duncan C. Gilbert, Mahesh K. B. Parmar, Nicholas D. James, Ruth E. Langley

Manuscript writing: All authors

Final approval of manuscript: All authors

Accountable for all aspects of the work: All authors

\section{ACKNOWLEDGMENT}

The authors would like to thank the patients and their families, site research staff, and members of the wider STAMPEDE trial management groups, trial steering committee, and independent data monitoring committee.

\section{REFERENCES}

1. Gillessen S, Attard G, Beer TM, et al: Management of patients with advanced prostate cancer: Report of the Advanced Prostate Cancer Consensus Conference 2019. Eur Urol 77:508-547, 2020

2. James ND, Sydes MR, Clarke NW, et al: Addition of docetaxel, zoledronic acid, or both to first-line long-term hormone therapy in prostate cancer (STAMPEDE): Survival results from an adaptive, multiarm, multistage, platform randomised controlled trial. Lancet 387:1163-1177, 2016

3. James ND, de Bono JS, Spears MR, et al: Abiraterone for prostate cancer not previously treated with hormone therapy. N Engl J Med 377:338-351, 2017

4. Sweeney CJ, Chen YH, Carducci M, et al: Chemohormonal therapy in metastatic hormone-sensitive prostate cancer. N Engl J Med 373:737-746, 2015

5. Fizazi K, Tran N, Fein L, et al: Abiraterone plus prednisone in metastatic, castration-sensitive prostate cancer. N Engl J Med 377:352-360, 2017

6. Vale CL, Burdett S, Rydzewska LHM, et al: Addition of docetaxel or bisphosphonates to standard of care in men with localised or metastatic, hormone-sensitive prostate cancer: A systematic review and meta-analyses of aggregate data. Lancet Oncol 17:243-256, 2016

7. Rydzewska LHM, Burdett S, Vale CL, et al: Adding abiraterone to androgen deprivation therapy in men with metastatic hormone-sensitive prostate cancer: $\mathrm{A}$ systematic review and meta-analysis. Eur J Cancer 84:88-101, 2017

8. Watson V, McCartan N, Krucien N, et al: Evaluating the trade-offs men with localised prostate cancer make between the risks and benefits of treatments: The COMPARE study. J Urol 204:273-280, 2020

9. Gilbert DC, Duong T, Kynaston HG, et al: Quality-of-life outcomes from the Prostate Adenocarcinoma: TransCutaneous Hormones (PATCH) trial evaluating luteinising hormone-releasing hormone agonists versus transdermal oestradiol for androgen suppression in advanced prostate cancer. BJU Int 119:667-675, 2017

10. Sydes MR, Spears MR, Mason MD, et al: Adding abiraterone or docetaxel to long-term hormone therapy for prostate cancer: Directly randomised data from the STAMPEDE multi-arm, multi-stage platform protocol. Ann Oncol 29:1235-1248, 2018

11. James ND, Sydes MR, Clarke NW, et al: STAMPEDE: Systemic therapy for advancing or metastatic prostate cancer-A multi-arm multi-stage randomised controlled trial. Clin Oncol (R Coll Radiol) 20:577-581, 2008

12. Aaronson NK, Ahmedzai S, Bergman B, et al: The European Organization for Research and Treatment of Cancer QLQ-C30: A quality-of-life instrument for use in international clinical trials in oncology. J Natl Cancer Inst 85:365-376, 1993

13. van Andel G, Bottomley A, Fossa SD, et al: An international field study of the EORTC QLQ-PR25: A questionnaire for assessing the health-related quality of life of patients with prostate cancer. Eur J Cancer 44:2418-2424, 2008 
14. Fayers PM, Aaronson NK, Bjordal K, et al: The EORTC QLQ-C30 Scoring Manual (ed 3). Brussels, Belgium, European Organisation for Research and Treatment of Cancer, 2001

15. Cocks K, King MT, Velikova G, et al: Evidence-based guidelines for determination of sample size and interpretation of the European Organisation for the Research and Treatment of Cancer Quality of Life Questionnaire Core 30. J Clin Oncol 29:89-96, 2011

16. Patrick DL, Burke LB, Powers JH, et al: Patient-reported outcomes to support medical product labeling claims: FDA perspective. Value Health 10:S125-S137, 2007 (suppl 2)

17. Calvert M, Blazeby J, Altman DG, et al: Reporting of patient-reported outcomes in randomized trials: The CONSORT PRO extension. JAMA 309:814-822, 2013

18. Berry DL, Moinpour CM, Jiang CS, et al: Quality of life and pain in advanced stage prostate cancer: Results of a Southwest Oncology Group randomized trial comparing docetaxel and estramustine to mitoxantrone and prednisone. J Clin Oncol 24:2828-2835, 2006

19. Tannock IF, de Wit R, Berry WR, et al: Docetaxel plus prednisone or mitoxantrone plus prednisone for advanced prostate cancer. N Engl J Med 351:1502-1512, 2004

20. Morgans AK, Chen YH, Sweeney CJ, et al: Quality of life during treatment with chemohormonal therapy: Analysis of E3805 chemohormonal androgen ablation randomized trial in prostate cancer. J Clin Oncol 36:1088-1095, 2018

21. Chi KN, Protheroe A, Rodriguez-Antolin A, et al: Patient-reported outcomes following abiraterone acetate plus prednisone added to androgen deprivation therapy in patients with newly diagnosed metastatic castration-naive prostate cancer (LATITUDE): An international, randomised phase 3 trial. Lancet Oncol 19: 194-206, 2018

22. Feyerabend S, Saad F, Li T, et al: Survival benefit, disease progression and quality-of-life outcomes of abiraterone acetate plus prednisone versus docetaxel in metastatic hormone-sensitive prostate cancer: A network meta-analysis. Eur J Cancer 103:78-87, 2018

23. Cocks K, King MT, Velikova G, et al: Evidence-based guidelines for interpreting change scores for the European Organisation for the Research and Treatment of Cancer Quality of Life Questionnaire Core 30. Eur J Cancer 48:1713-1721, 2012

24. Atkinson TM, Wagner J-S, Basch E: Trustworthiness of patient-reported outcomes in unblinded cancer clinical trials. JAMA Oncol 3:738-739, 2017

25. Mouillet G, Efficace F, Thiery-Vuillemin A, et al: Investigating the impact of open label design on patient-reported outcome results in prostate cancer randomized controlled trials. Cancer Med 9:7363-7374, 2020

26. Roydhouse JK, King-Kallimanis BL, Howie LJ, et al: Blinding and patient-reported outcome completion rates in US Food and Drug Administration Cancer Trial Submissions, 2007-2017. J Natl Cancer Inst 111:459-464, 2018

27. Fielding S, Maclennan G, Cook JA, et al: A review of RCTs in four medical journals to assess the use of imputation to overcome missing data in quality of life outcomes. Trials 9:51, 2008

28. Bell ML, King MT, Fairclough DL: Bias in area under the curve for longitudinal clinical trials with missing patient reported outcome data: Summary measures versus summary statistics. SAGE Open doi:10.1177/2158244014534858

29. Sullivan TR, White IR, Salter AB, et al: Should multiple imputation be the method of choice for handling missing data in randomized trials? Stat Methods Med Res 27:2610-2626, 2018 


\section{AUTHORS' DISCLOSURES OF POTENTIAL CONFLICTS OF INTEREST}

\section{Quality of Life in Men With Prostate Cancer Randomly Allocated to Receive Docetaxel or Abiraterone in the STAMPEDE Trial}

The following represents disclosure information provided by authors of this manuscript. All relationships are considered compensated unless otherwise noted. Relationships are self-held unless noted. I = Immediate Family Member, Inst = My Institution. Relationships may not relate to the subject matter of this manuscript. For more information about ASCO's conflict of interest policy, please refer to www.asco.org/rwc or ascopubs.org/jco/authors/author-center.

Open Payments is a public database containing information reported by companies about payments made to US-licensed physicians (Open Payments).

\section{Alicia K. Morgans}

Honoraria: Genentech, Janssen, Sanofi, AstraZeneca, Astellas Scientific and Medical Affairs Inc, Astellas Pharma, Janssen Oncology, Bayer, Janssen, Clovis Oncology, Myovant Sciences, Advanced Accelerator Applications, Exelixis, Pfizer, Merck

Consulting or Advisory Role: AstraZeneca, Sanofi, Bayer, Astellas Pharma, Janssen, Advanced Accelerator Applications, Myovant Sciences, Blue Earth Diagnostics, Myovant Sciences, Exelixis, Novartis, Myriad Genetics, Lantheus Medical Imaging, Merck

Research Funding: Bayer, Seattle Genetics/Astellas, Genentech, AstraZeneca Astellas Scientific and Medical Affairs Inc, Dendreon, Sanofi, Myovant Sciences Travel, Accommodations, Expenses: Sanofi

Noel W. Clarke

Honoraria: Janssen-Cilag, Astellas Pharma, Sanofi, Bayer, AstraZeneca, Ferring, Ipsen

Consulting or Advisory Role: Astellas Pharma, Janssen-Cilag, Ferring, Bayer, Sanofi

Speakers' Bureau: Janssen-Cilag, Astellas Pharma

Research Funding: Astrazeneca

Travel, Accommodations, Expenses: Janssen-Cilag, Astellas Pharma, Sanofi, Bayer, AstraZeneca, Ferring, Ipsen

\section{Gerhardt Attard}

Honoraria: Astellas Pharma, Janssen (I)

Consulting or Advisory Role: Janssen-Cilag, Veridex, Ventana Medical Systems, Astellas Pharma, Medivation, Novartis, Millennium, Abbott Laboratories, ESSA Bayer, Pfizer, AstraZeneca, Ferring

Speakers' Bureau: Janssen, Astellas Pharma, Takeda, Sanofi, Ventana Medical Systems, Ipsen, AstraZeneca, Ferring

Research Funding: Janssen, Arno Therapeutics, Innocrin Pharma

Patents, Royalties, Other Intellectual Property: I am on The ICR rewards to inventors list of abiraterone acetate

Travel, Accommodations, Expenses: Astellas Pharma, Medivation, Ventana Medical Systems, Abbott Laboratories, Bayer, ESSA, Janssen (I), Astellas Pharma (I), Pfizer (I), Ferring

Other Relationship: Institute of Cancer Research

David P. Dearnaley

Patents, Royalties, Other Intellectual Property: Abiraterone acetate was developed at The Institute of Cancer Research, which therefore has a commercial interest in the development of this agent. Professor Dearnaley is on the Institute's Rewards to Inventors list for abiraterone acetate, patent issued EP1933709B1 (location and stabilization device)

Travel, Accommodations, Expenses: Janssen Oncology

Christopher C. Parker

Honoraria: Bayer, Janssen

Consulting or Advisory Role: Clarity Pharmaceuticals, Myovant Sciences, ITM Oncologics

Silke Gillessen

Consulting or Advisory Role: Astellas Pharma (Inst), Janssen (Inst), Bayer (Inst) Orion Pharma GmbH, Tolero Pharmaceuticals (Inst), MSD Oncology (Inst), Roche (Inst), Amgen, Pfizer (Inst), BMS (Inst), Telix Pharma (Inst), MSD

Oncology, AAA HealthCare (Inst), Orion Corporation (Inst), Amgen (Inst)

Patents, Royalties, Other Intellectual Property: Method for biomarker (WO 3752009138392 A1)

Travel, Accommodations, Expenses: ProteoMedix

Other Relationship: ProteoMedix, Aranda Pharma (Inst), Menarini Silicon

Biosystems (Inst)

Christopher D. Brawley

Research Funding: Astellas Pharma, Clovis Oncology, Janssen, Sanofi/Aventis, Pfizer, Novartis,

Jacob S. Tanguay

Consulting or Advisory Role: AstraZeneca, Astellas Pharma, Bayer

Travel, Accommodations, Expenses: Janssen, Roche, Bayer
Robert J. Jones

Honoraria: Astellas Pharma, Janssen, AstraZeneca, MSD Oncology, Bristol

Myers Squibb, Pfizer, Novartis, Ipsen, Seattle Genetics, Sanofi, Bayer, Roche/ Genentech, EUSA Pharma, Pharmacyclics, Clovis Oncology

Research Funding: Roche, Astellas Pharma, AstraZeneca, Pfizer, Novartis,

Exelixis, Clovis Oncology, Bayer

Travel, Accommodations, Expenses: Ipsen, Bayer, Janssen, Astellas Pharma, MSD

\section{John Wagstaff}

Honoraria: Bristol Myers Squibb, Roche, Pierre Fabre, Novartis,

GlaxoSmithKline UK Ltd., Pfizer

Consulting or Advisory Role: Bristol Myers Squibb, Roche, Pfizer, Novartis, Pierre Fabre

Speakers' Bureau: Bristol Myers Squibb

Sarah Rudman

Honoraria: Bristol Myers Squibb Foundation, EUSA Pharma, Ipsen, Pfizer Consulting or Advisory Role: Ipsen, Pfizer, EUSA Pharma

Joe M. O'Sullivan

Consulting or Advisory Role: Bayer, Janssen, Astellas Pharma, Sanofi, Novartis Speakers' Bureau: Bayer, Janssen, Novartis

Research Funding: Bayer

\section{Alison Birtle}

Honoraria: Janssen-Cilag

Consulting or Advisory Role: Roche, Astellas Medivation, Janssen Oncology, AstraZeneca, Sanofi, Bayer Schering Pharma, Bristol Myers Squib, Merck

Serono, Pfizer

Speakers' Bureau: Bayer, Janssen Oncology, Pfizer

Research Funding: Genzyme

Andrew Protheroe

Employment: Genesis Cancer Care

Honoraria: Astellas Pharma, MSD Oncology, Ipsen, Astellas Pharma, Merck Serono, Eisai, Merck

Travel, Accommodations, Expenses: Bayer, EUSA Pharma

Emma Gray

Employment: Genesis Cancer Care

Honoraria: Astellas Pharma, MSD Oncology, Ipsen, Astellas Pharma, Merck Serono, Eisai, Merck

Travel, Accommodations, Expenses: Bayer, EUSA Pharma

Neil McPhail

Consulting or Advisory Role: Eisai, GlaxoSmithKline, Ipsen

Travel, Accommodations, Expenses: Ipsen

Zaf I. Malik

Consulting or Advisory Role: Sanofi/Aventis

Salil Vengalil

Honoraria: Bristol Myers Squibb, Pfizer

Consulting or Advisory Role: Merck/Pfizer

Travel, Accommodations, Expenses: Ipsen

Peter Hoskin

Research Funding: Varian Medical Systems, Astellas Pharma, Bayer, Roche, Pfizer, Elekta

Matthew R. Sydes

Honoraria: Lilly, Sanofi, Janssen

Research Funding: Astellas Pharma, Janssen-Cilag, Pfizer, Novartis, Sanofi, Clovis Oncology

Simon Chowdhury

Honoraria: Clovis Oncology, Novartis

Consulting or Advisory Role: Clovis Oncology, Astellas Pharma, Bayer, Pfizer, Janssen-Cilag, BeiGene, Novartis

Speakers' Bureau: Pfizer, Janssen-Cilag

Research Funding: Sanofi/Aventis

Mahesh K. B. Parmar

Research Funding: AstraZeneca, Astellas Pharma, Janssen, Clovis Oncology 
Nicholas D. James

Honoraria: Sanofi, Bayer, Janssen, Astellas Pharma

Consulting or Advisory Role: Sanofi, Bayer, Astellas Pharma, Janssen, Clovis Oncology, EUSA pharma, pfizer

Speakers' Bureau: Pierre Fabre, Ferring, Sanofi, Astellas Pharma, Janssen Oncology, Merck

Research Funding: Janssen, Astellas Pharma, Pfizer, Sanofi, Novartis,

AstraZeneca

Travel, Accommodations, Expenses: Sanofi, Janssen
Ruth E. Langley

Consulting or Advisory Role: Bayer Schering Pharma Research Funding: Bayer Schering Pharma

No other potential conflicts of interest were reported. 


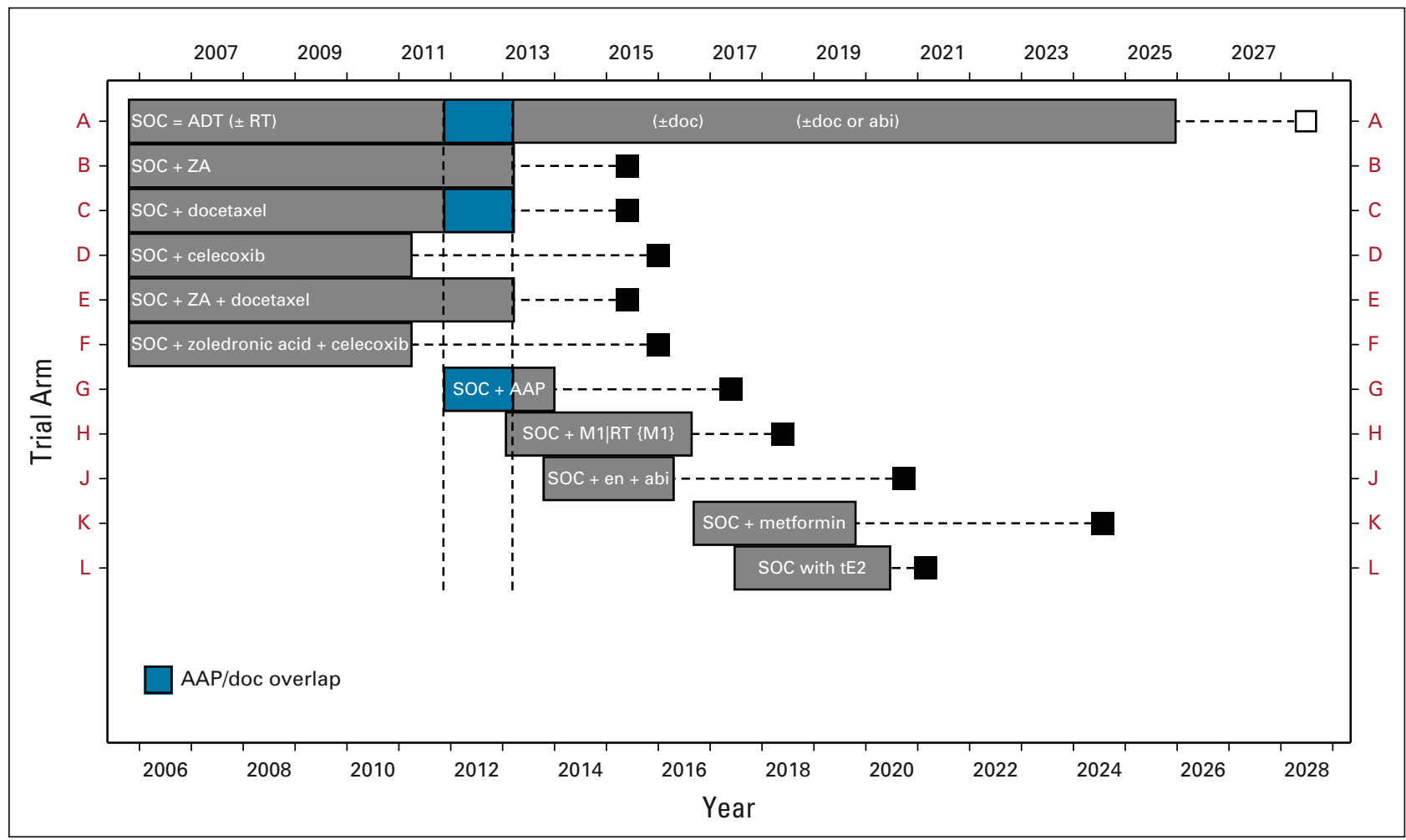

FIG A1. Activity by time in the STAMPEDE trial. Gray boxes represent periods of recruitment ( $x$-axis) to each of the trial arms ( $y$-axis). The black squares represent the timepoint of the first key comparative analysis for each trial arm. Patients included in this QOL analysis were randomly assigned during the period highlighted in blue; the primary analysis is between docetaxel + SOC and AAP + SOC, with SOC patients presented for context. AAP, abiraterone acetate plus prednisone or prednisolone; abi, abiraterone; doc, docetaxel; en, enzalutamide; M1, metastatic prostate cancer; QOL, quality of life; SABR, stereotactic ablative radiotherapy; SOC, standard of care; tE2, transdermal oestradiol; ZA, zoledronic acid. 


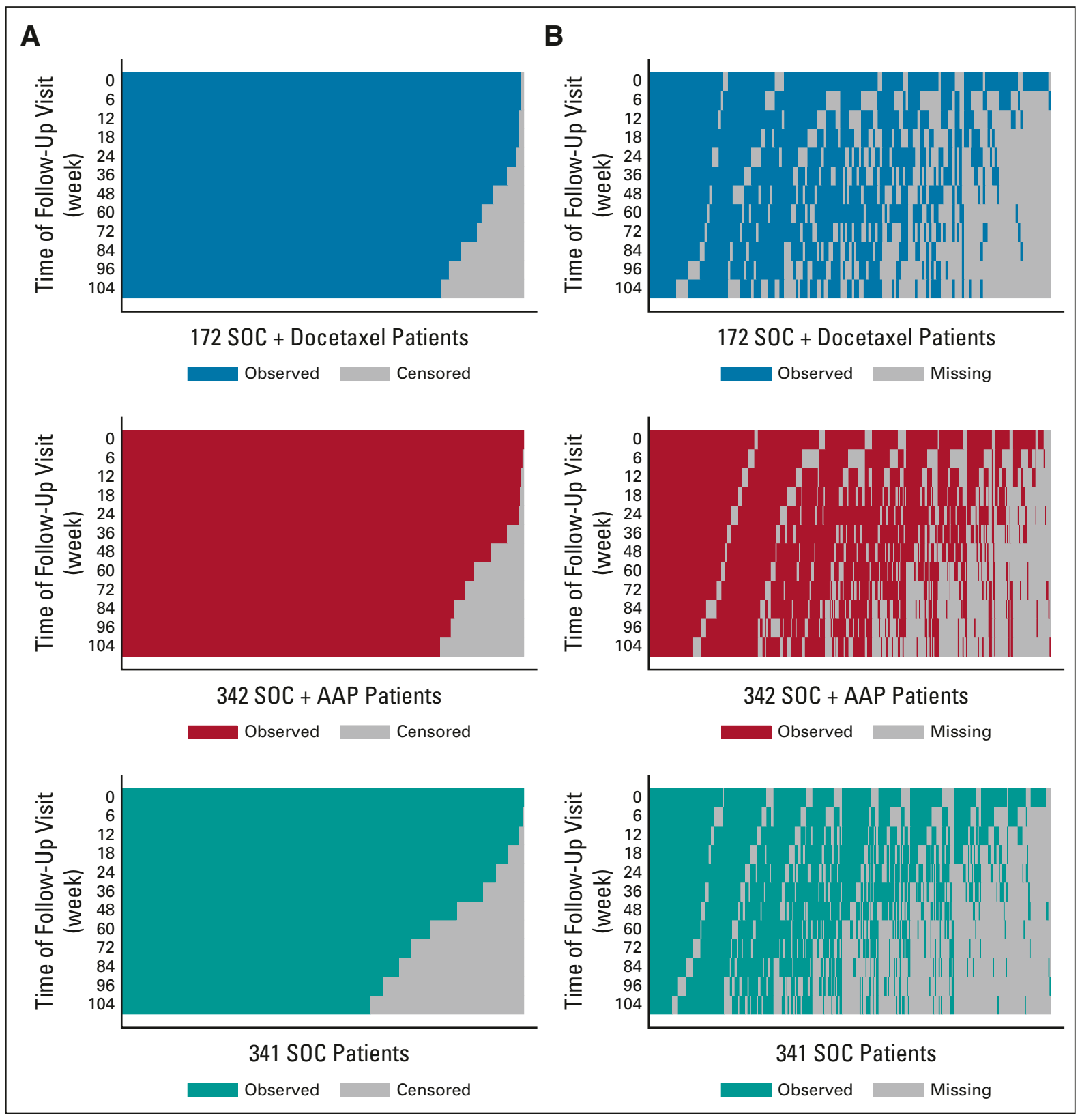

FIG A2. Patterns of missing patient data. (A) Censored data presented per allocated treatment. Each row represents a follow-up appointment where we would expect the patient to complete a QOL questionnaire. Each column represents a patient. A solid color indicates the patient was considered in this analysis. Gray means the patient was censored for starting second-line treatment or death and any data received did not contribute to the analysis. (B) Missing data presented by allocated treatment. Each row represents a follow-up appointment where we would expect the patient to complete a QOL questionnaire. Each column represents a patient. A solid color indicates the patient provided a QOL questionnaire at this timepoint. Gray means there was no QOL questionnaire received and reflects missing data from the analysis. AAP, abiraterone acetate plus prednisolone or prednisone; QOL, quality of life. 


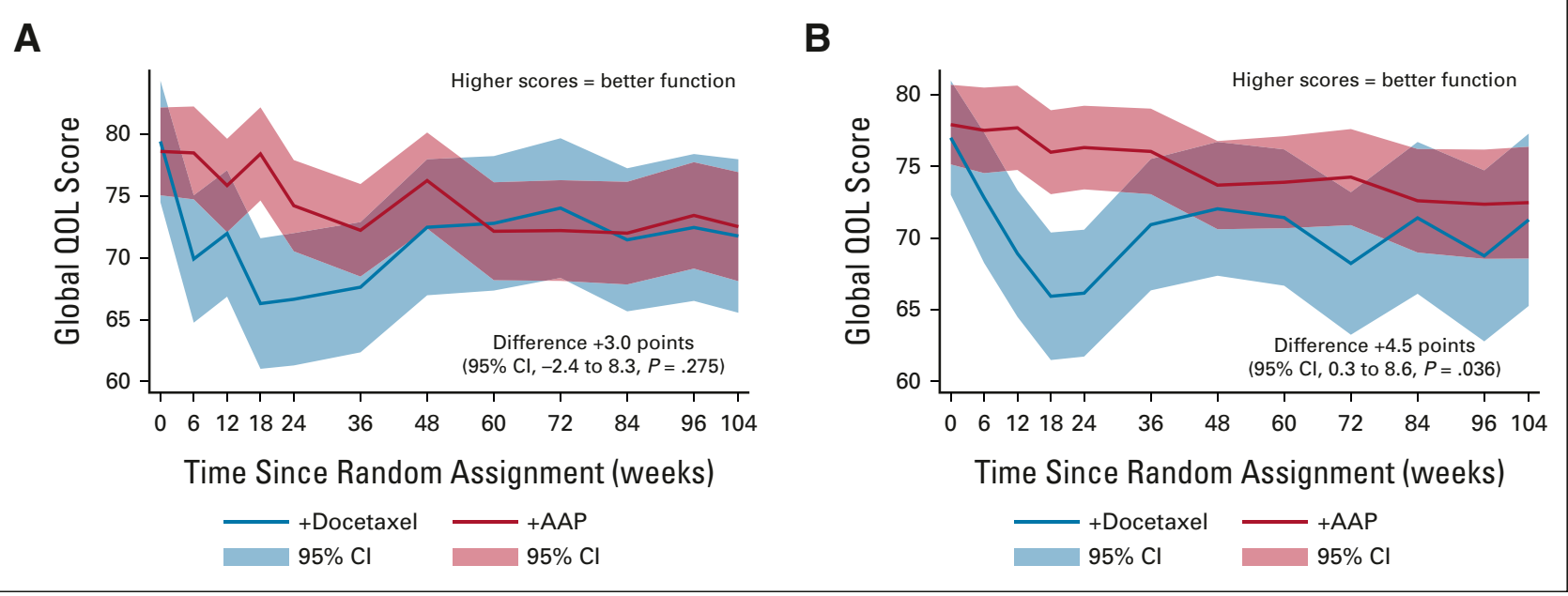

FIG A3. Longitudinal analysis showing global-QOL score over the first 2 years in patients with nonmetastatic disease and metastatic disease, separating scores for patients treated with docetaxel + SOC versus AAP + SOC. The difference is calculated by subtracting the docetaxel + SOC score from the AAP + SOC score; a positive difference represents a higher QOL for patients treated with AAP + SOC and a negative difference indicates a higher QOL for patients receiving docetaxel + SOC. Higher scores indicate better QOL. All scores are corrected as per QLQ-C30 guidelines to range from 0 to 100: (A) nonmetastatic disease and (B) metastatic disease. AAP, abiraterone acetate plus prednisone or prednisolone; QOL, quality of life; SOC, standard of care.

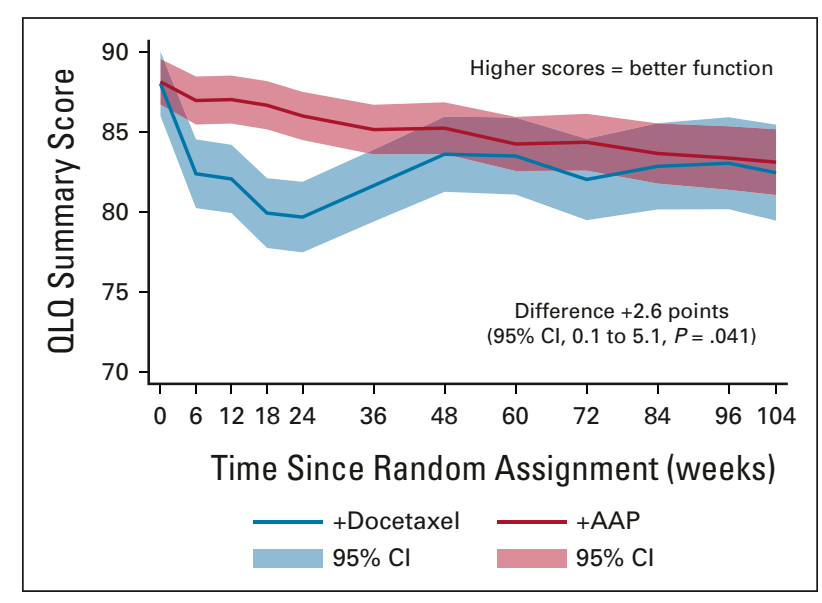

FIG A4. Longitudinal analysis showing the QLQ-C30 summary score over the first 2 years, separating scores for patients treated with docetaxel $+\mathrm{SOC}$ versus AAP $+\mathrm{SOC}$. The difference is calculated by subtracting the docetaxel + SOC score from the AAP + SOC score; a positive difference represents a higher $\mathrm{QOL}$ for patients treated with $\mathrm{AAP}+\mathrm{SOC}$ and a negative difference indicates a higher $\mathrm{QOL}$ for patients receiving docetaxel + SOC. Higher scores indicate better quality of life. All scores are corrected as per QLQ-C30 guidelines to range from 0 to 100 . AAP, abiraterone acetate plus prednisone or prednisolone; QOL, quality of life; SOC, standard of care.

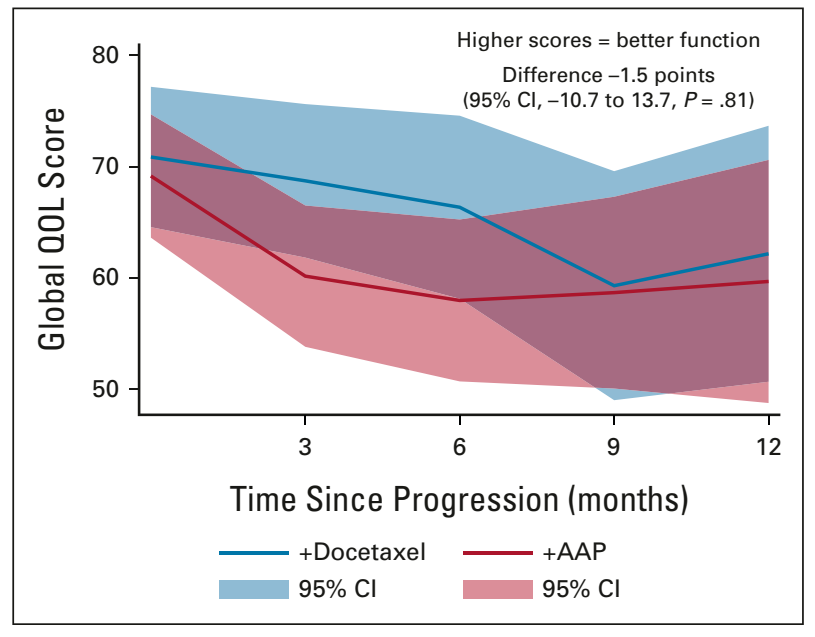

FIG A5. Longitudinal analysis showing the global-QOL score that occurred after progression, separating scores for patients treated in the upfront setting with docetaxel $+\mathrm{SOC}$ and $\mathrm{AAP}+\mathrm{SOC}$ (ie, postprogression patients are potentially now receiving a different subsequent treatment). The difference is calculated by subtracting the docetaxel + SOC score from the AAP + SOC score; thus, positive a difference represents a higher $\mathrm{QOL}$ for patients treated with $\mathrm{AAP}+\mathrm{SOC}$ and a negative difference indicates a higher $\mathrm{QOL}$ for patients treated with docetaxel + SOC. Higher scores indicate better quality of life. All scores are corrected as per QLQ-C30 guidelines to range from 0 to 100 . AAP, abiraterone acetate plus prednisone or prednisolone; QOL, quality of life; SOC, standard of care. 
TABLE A1. Suggested Boundaries for Determining Magnitude of Difference in QOL Scores and Whether a Difference is Clinically Meaningful: Small, Moderate, and Large Differences Are all Considered Potentially Clinically Meaningful

Boundary

Trivial

Small

Moderate

Large

\begin{tabular}{llllr}
\hline Global-QOL & $0-4$ & $4-10$ & $10-15$ & $14-22$ \\
\hline Physical functioning & $0-5$ & $5-14$ & $11-15$ & $>22$ \\
\hline Social functioning & $0-5$ & $5-11$ & $19-29$ & -15 \\
\hline Role functioning & $0-6$ & $6-19$ & $3-14$ & $13-19$ \\
\hline Cognitive functioning & $0-3$ & $6-13$ & $13-19$ & $>14$ \\
\hline Pain & $0-6$ & $5-13$ & $>19$
\end{tabular}

NOTE. This table has been adapted from Cocks et al. ${ }^{15}$

Abbreviation: QOL, quality of life.

TABLE A2. Proportion of Missing QOL Data at Each Timepoint for Patients in Treated With SOC, Docetaxel + SOC, and AAP + SOC

\begin{tabular}{|c|c|c|c|c|c|c|c|c|c|}
\hline \multirow[b]{2}{*}{ Week } & \multicolumn{3}{|c|}{ SOC } & \multicolumn{3}{|c|}{ Docetaxel + SOC } & \multicolumn{3}{|c|}{ AAP + SOC } \\
\hline & Received & Expected & $\%$ & Received & Expected & $\%$ & Received & Expected & $\%$ \\
\hline 0 & 283 & 341 & 83 & 153 & 171 & 89 & 303 & 342 & 89 \\
\hline 12 & 245 & 336 & 73 & 116 & 170 & 68 & 249 & 340 & 73 \\
\hline 18 & 226 & 327 & 69 & 109 & 170 & 64 & 248 & 339 & 73 \\
\hline 36 & 223 & 306 & 73 & 109 & 165 & 66 & 253 & 328 & 77 \\
\hline 48 & 204 & 284 & 72 & 106 & 159 & 67 & 241 & 314 & 77 \\
\hline 60 & 183 & 261 & 70 & 111 & 154 & 72 & 229 & 300 & 76 \\
\hline 72 & 164 & 245 & 67 & 103 & 152 & 68 & 216 & 292 & 74 \\
\hline Total & 2,412 & 3,424 & 70 & 1,283 & 1,903 & 67 & 2,809 & 3,768 & 75 \\
\hline
\end{tabular}

NOTE. The expected denominator reflects all patients who had not been censored (patients were censored at start of second-line treatment or death) at each timepoint. Received is the number of QOL questionnaires received at each timepoint.

Abbreviations: AAP, abiraterone acetate plus prednisone or prednisolone; QOL, quality of life; SOC, standard of care. 